\title{
MANAGEMENT AND TRANSCENDENCE OF INCIDENTAL THYROID 18FDG-PET FOCAL UPTAKE (PEToma)
}

Anna Casteràs Roman ${ }^{1}$, Carles Zafon Llopis ${ }^{1}$, Amparo García Burillo², Diego Villasboas Rosciolesi', Maite Salcedo Pujanell ${ }^{2}$, Jaime Monturiol Duran ${ }^{2}$, Joan Castell Conesa ${ }^{2}$, Jordi Mesa Manteca ${ }^{1}$.

${ }^{1}$ Endocrinology Department, Universitary Hospital Vall d'Hebron, Barcelona, Spain
${ }^{2}$ Department of Nuclear Medicine, Universitary Hospital Vall d'Hebron, Barcelona, Spain

INTRODUCTION: Incidental focal uptake in the thyroid gland is detected in 1\%-2\% of 18FDG-PET examinations. FNA is recommended due to an increased risk of malignancy (25-50\%). However, these figures are extracted from the studied cases, which are only about a half of all thyroid PETomas. Moreover there is controversy about the maximum standardized uptake value (SUV $\left.\mathrm{V}_{\text {max }}\right)$ ability to discriminate between benign or malignant nodules.

METHODS: We retrospectively reviewed 4207 FDG-PET scans performed for non-thyroidal reasons from January 2013 to October

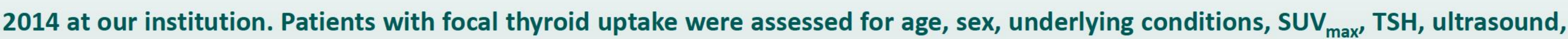
FNA cytology and pathological findings.

\section{RESULTS:}

$>65$ cases (1,54\% of PETs) showed focal thyroid uptake, 41 (63\%) females, median age (SD) $70(12)$ years.

$>$ Median (SD) SUV $\max$ was 7.4 (6), [1.4- 32].

$>$ In global 49 patients (75.4 \%) were found to have cancer, most common lung cancer and lymphoma, and currently 19 patients (29.2\%) have died.

- Ultrasound was performed in 36 patients (55,4\%): single nodule 16 , multinodular 19, normal 1; mean nodular size was 16.6 (9.6) $\mathrm{mm}$; in 9 cases the nodule was suspictious.

$>$ Twenty-five cases (38.5\%) underwent FNA.

Bethesda score was benign (II) in $40 \%$, followed by insufficient sample (24\%) and malignant (VI) in $16 \%$ of cytologies.

Seven patients were operated on, 5 were thyroid cancer. ( Fig 1)
Fig 1. Thyroid PEToma flowchart

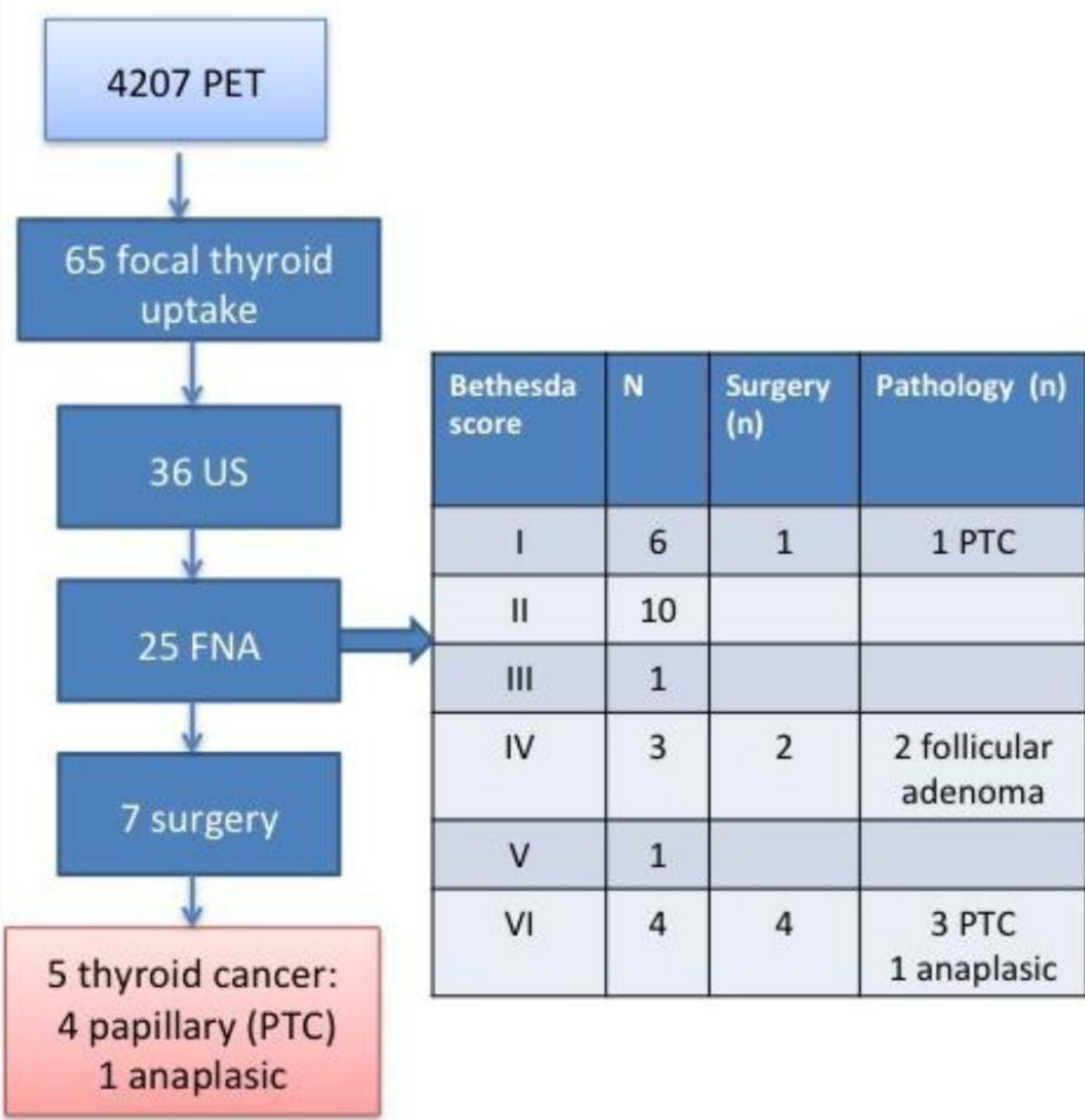

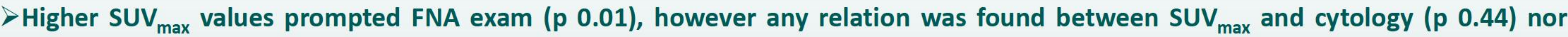
nodular size (p 0.07).

$>$ There was a direct correlation between TSH levels and $\operatorname{SUV}_{\max }(p<0.001)$, but disappeared when analizing euthyroidal patients $(\mathrm{TSH}$ $0.5-4.5, n=53)$ p 0.9

We report similar frequencies and clinical workup of thyroid PEToma compared to previous series.

Few cases underwent thyroidectomy, however thyroid cancer was a frequent histological finding.

SUV $_{\max }$ could not determine risk of malignancy. 